\title{
BMJ Open Rotating night shift work, sleep duration and elevated gamma-glutamyl transpeptidase among steelworkers: cross-sectional analyses from a Chinese occupational cohort
}

To cite: Li Q, Zhang S, Wang $\mathrm{H}$, et al. Rotating night shift work, sleep duration and elevated gamma-glutamyl transpeptidase among steelworkers: cross-sectional analyses from a Chinese occupational cohort. BMJ Open 2021;11:e053125. doi:10.1136/ bmjopen-2021-053125

- Prepublication history and additional supplemental material for this paper are available online. To view these files, please visit the journal online (http://dx.doi.org/10.1136/ bmjopen-2021-053125)

QL and SZ contributed equally.

Received 05 May 2021

Accepted 22 November 2021

Check for updates

(c) Author(s) (or their employer(s)) 2021. Re-use permitted under CC BY-NC. No commercial re-use. See rights and permissions. Published by BMJ.

School of Public Health, North China University of Science and Technology, Tangshan, Hebei, China

Correspondence to Dr Juxiang Yuan; jxyuan5082@163.com

\section{ABSTRACT}

Objectives This study aimed to evaluate the separate and combined effects of rotating night shift work and lifestyle factors with elevated gamma-glutamyl transpeptidase (GGT) among steelworkers.

Design, setting and participants This cross-sectional study used the baseline information from a Chinese occupational cohort. The in-service workers of the production department of Tangsteel Company who participated in the occupational health examination in Tangshan from February to June 2017 were selected as the research objects.

Main outcome measures The separate and combined effects of rotating night shift work and lifestyle factors with elevated GGT among steelworkers.

Results The information of 7031 subjects from the production department of Tangsteel Company was analysed. Results showed that the current shift workers and the workers with the duration of night shifts $>19$ years, the cumulative number of night shifts $>1774$ nights, the average frequency of night shifts $\leq 7$ nights/month and the average frequency of night shifts $>7$ nights/month had elevated odds of elevated GGT, compared with those who never worked night shifts, and ORs, $(95 \% \mathrm{Cls})$ were 1.39 , (1.10 to 1.75$), 1.46$, (1.15 to 1.86$), 1.46,(1.15$ to 1.85), 1.34, (1.04 to 1.73) and 1.37, (1.09 to 1.74) after adjustment for potential confounders. The independent effect of shorter sleep duration ( $<7$ hours/day) on elevated GGT was not statistically significant. Among workers who had shorter sleep duration, the association between rotating night shift work and elevated GGT was statistically significant, but no associations were found among workers with the sleep duration of $\geq 7$ hours/day. In addition, other lifestyle factors affected the association between rotating night shift work and elevated GGT.

Conclusions Rotating night shift work is associated with elevated GGT among steelworkers. In particular, the effect of rotating night shift work on elevated GGT was affected by sleep duration and other lifestyle factors.

\section{INTRODUCTION}

Shift work refers to irregular and abnormal working time arrangements, including all
Strengths and limitations of this study

This study contains a large sample size and rich information (risk factors in the occupational environment, detailed disease history, general demographic characteristics and lifestyle information) of subjects.

- In this study, different exposure indicators of night shift work were used to evaluate rotating night shift work, and this is the first study to explore the combined effect of sleep duration and rotating night shift work on liver enzymes.

- We have no authority to obtain the specific level of occupational hazards, including high temperature, noise, dust and carbon monoxide, so we were unable to analyse the effect of industrial dust in this study.

- This study is a cross-sectional study, which has limitations in explaining the causal relationship of rotating night shift work and sleep duration with elevated gamma-glutamyl transpeptidase.

working hours that are outside the normal daytime ones, ${ }^{1}$ such as rotating night shift work. Nowadays, rotating night shift work widely exists in the service industry, manufacturing industry, medicine industry and so on, and shift workers are required to work according to different schedules. ${ }^{2}$ It is reported that rotating night shift work is associated with many adverse health outcomes. ${ }^{3-5}$ Rotating night shift work will disrupt the synchronisation of the endogenous environment and the external environment by breaking the original sleep/wake cycle, thus affecting the secretion of hormones and the function of the circadian rhythm system and finally endangers human health. ${ }^{6-8}$ However, there are other lifestyles that affect circadian rhythm. Sleep is an essential part of lifestyle, and lack of sleep duration is associated with 
many adverse health outcomes. ${ }^{9-11}$ Studies have shown that insufficient sleep duration is associated with adverse outcomes of liver function. ${ }^{12}$

Gamma-glutamyl transpeptidase (GGT) is abundant in the liver and also present in the kidney, pancreas, which is one of the indicators of liver function detection. ${ }^{13}$ As a biochemical marker of hepatobiliary injury, the elevated GGT level is associated with pancreatic disease, fatty liver, renal failure and hepatobiliary disease. ${ }^{14-16}$

Some studies have shown that in addition to higher hyperhomocysteinaemia (HHcy) odds, ${ }^{8}$ rotating night shift work could be associated with adverse effects on liver function through circadian rhythm disorder and metabolic dysfunctions by redistributions of food consumption. ${ }^{17-19}$ Previous studies have shown that rotating night shift work is significantly associated with adverse reactions of liver function and higher levels of liver enzymes (such as alanine transaminase (ALT) and alkaline phosphatase (ALP)). ${ }^{20} 21$ However, the relationship between rotating night shift work and the level of GGT is not clear so far, and there are few related studies. In some studies, the evaluation indicators of night shift work (yes or no) ignored the important factors that may have an impact on health, such as the duration of night shifts (years) and the average frequency of night shifts (night/month), and thus concealing the real relationship between exposures and outcomes. In addition, rotating night shift work is not only related to light exposure at night, but also to sleeping duration and other lifestyle factors, and shift workers are known to sleep less than day workers. ${ }^{22}$ To our knowledge, no studies have focused on the combined effects of sleep duration and rotating night shift work on the level of GGT. This study aimed at exploring the relationship between rotating night shift work and elevated GGT with some indicators we have used, including duration of night shifts (years), cumulative number of night shifts (nights), average frequency of night shifts (night/ month) and current shift status. In particular, we also investigated the joint effects of some lifestyle factors and rotating night shift work on elevated GGT.

\section{METHODS \\ Study population}

Our data comes from a cohort of occupational health effects approved by the Ministry of science and technology of China. The purpose of this cohort study is to study the impact of some occupational harmful factors on human health. The cohort study was conducted in HBIS Group's Tangsteel Company in Tangshan city, China, and the baseline information of the cohort study was used in this study. The in-service workers in the production department of Tangsteel Company who participated in the occupational health examination in Tangshan Hongci hospital from February to June 2017 were selected as the research objects and the data used in previous publication $^{8}$ and this study was collected from the same participants. The workers with missing blood biochemical data, shift information and covariates, as well as those taking some antiretroviral drugs, and the workers with cancer, excess alcohol intake, history of hepatobiliary disease, thyroid disease and renal failure were excluded. A total of 7661 people participated in the occupational health examination, and finally, 7031 people were included in the study. All participants gave informed consent before taking part in this study.

\section{Patient and public involvement}

It was not appropriate or possible to involve patients or the public in the design, or conduct, or reporting, or dissemination plans of our research.

\section{Blood tests and elevated GGT}

Steelworkers were required to fast for at least 8 hours before their blood can be drawn. Because the blood index of workers who have just left the night shift may change briefly, and night shift workers need to be drawn on rest days. Participants' anterior elbow vein blood was collected and centrifuged at room temperature $(3000 \mathrm{r} /$ min, $15 \mathrm{~min}$ ) immediately. All blood samples were tested in the central laboratory of Tangshan Hongci Hospital using automatic biochemical analysers (mindray, BS-800, China) within 4 hours. Elevated ALT and elevated aspartate aminotransferase (AST) were defined as ALT $>40 \mathrm{U} / \mathrm{L}$ and $\mathrm{AST}>40 \mathrm{U} / \mathrm{L}$, respectively. Elevated GGT was defined as: (GGT $>60 \mathrm{U} / \mathrm{L}$ in males, GGT $>40 \mathrm{U} / \mathrm{L}$ in females) according to the reference value of the central laboratory of Tangshan Hongci Hospital and other studies. ${ }^{23}$

\section{Assessment of rotating night shift work}

Shift work (the mainly four-crew-three-shift system now and historical three-crew-two-shift system) in this study refered to rotating night shift work. Shift workers whose shift duration did not exceed 1 year and the workers who worked regular working hours at all times were defined as never rotating night shift workers. In the four-crewthree-shift system, each group has two morning shifts (08:00-16:00), two afternoon shifts (16:00-00:00), two night shifts (00:00-08:00) and then has 2 days off. In the three-crew-two-shift system, each group has a morning shift (08:00-20:00), a night shift (20:00-08:00) and then takes 1 day off. The data were collected from face-to-face interviews and then checked with the Tangsteel Company's records. Participants were asked whether they were involved in rotating night shift work (working through 00:00 to 6:00) during their employment. If yes, they were asked about their current shift status (ever, current), shift system, number of days in each month for each shift system, start date and end date of each shift system. In this study, different exposure indicators were used to assess rotating night shift work. Duration of night shifts (years): sum of years spent in all different night shift systems; cumulative number of night shifts (nights): sum of nights spent in all different night shift systems; average frequency of night shifts (nights/month): cumulative number of night shifts (nights) divided by cumulative 
number of months of employment. The difference from previous publications is that the different exposure indicators of night shift work of continuous variables of this study are grouped according to the median. ${ }^{8}$

\section{Assessment of sleep duration}

Participants were asked how many hours of sleep each day when they go to different shifts (morning shift, afternoon shift and night shift) and how many hours of sleep each day when they rest. Sleep duration was the weighted mean of sleep duration on working days and non-working days. In the four-crew-three-shift system, sleep duration = [ (sleep duration of morning shift $\times 2)+$ (sleep duration of afternoon shift $\times 2)+($ sleep duration of night shift $\times 2)+$ (sleep duration at rest $\times 2$ ) ]/8. In the three-crew-two-shift system, sleep duration $=[$ (sleep duration of morning shift $\times 1)+($ sleep duration of night shift $\times 1)+($ sleep duration at rest $\times 1)] / 3$. For workers who never work shifts, sleep duration was a weighted average of the working and non-working days of a work/rest cycle. Sleep duration $<7$ hours/day was defined as shorter sleep duration.

\section{Questionnaire and assessment of covariates}

After the presurvey and expert consultation, the questionnaire was revised repeatedly before the formal use. All of the investigators received professional training in advance and conducted face-to-face interviews with the research objects by unified standards and methods to collect data. The contents of the questionnaire included sex, age, sleep, smoking and drinking status, physical activity, diet, rotating night shift work information and personal disease history (see online supplemental file).

\section{Statistical analyses}

Continuous variables were described by means \pm SD and median and range (minimum, maximum), and the differences between groups were compared by independent sample t-test if the data was normally distributed. Otherwise, the rank-sum test was used to compare these continuous variables among the various groups. The categorical variables were expressed by the number of individuals $(\%)$, and $\chi^{2}$ tests were used for comparison between groups. Multivariate unconditional logistic regression model and restricted cubic spline function (RCS) model were used to explore the relationship between different exposure indicators of night shift work and elevated GGT. For multivariate logistic regression, stepwise regression was used to select covariates. Analyses were conducted with Statistical Package for the Social Sciences (SPSS for Windows, V.19.0) software and Statistical Analysis System V.9.4 (SAS, Institute) with a two-sided significance threshold of $\mathrm{p}<0.05$.

\section{RESULTS}

Univariate analysis

Table 1 shows the basic characteristics of 7031 subjects according to current shift status. The difference between the different current shift status groups were statistically significantly by age, sex, working years and diabetes history. The range of age and working years of the subjects is (22.3-60.0) and (0.2-46.8), respectively. In terms of lifestyle, smoking status was statistically different among the different current shift status groups. In terms of occupational hazardous factors, exposure of high temperature, dust and carbon monoxide (CO) differed significantly among the different current shift status groups, but the differences were not statistically significant in different noise exposure groups. GGT levels and ALT levels differed significantly among the different current shift status groups, and current night shift workers were more likely to have elevated GGT and elevated ALT.

The differences in means of GGT according to current shift status were statistically significant (see online supplemental table S1). The higher exposure categories of night shift work showed an increased prevalence of elevated GGT (table 2). Subjects with elevated GGT had higher age and working years. And other basic characteristics of the study subjects according to GGT levels status were shown in online supplemental table S2.

\section{Multivariate analysis}

RCS models were used to fit the relationship between different exposure metrics of night shift work (duration of night shifts, cumulative number of night shifts) and elevated GGT (figures 1 and 2). After adjusting for age, sex, smoking status, drinking status and Dietary Approaches to Stop Hypertension (DASH) Score, the RCS curves were obtained. According to the Akaike information criterion and Bayesian information criterion, RCS curves fitted by five nodes were selected, and there were positive association between the different exposure metrics of night shift work and the prevalence of elevated GGT.

ORs of elevated GGT according to different exposure metrics of night shift work and sleep duration were shown in table 3. The relationship between current shift status, duration of night shifts, cumulative number of night shifts, average frequency of night shifts and elevated GGT were statistically significant. First, the results of model 1 showed that the odds of elevated GGT were increased with the groups of the current night shift, the duration of night shifts $>19$ years, the cumulative number of night shifts $>1774$ nights, the average frequency of night shifts $\leq 7 \mathrm{night} /$ month and the average frequency of night shifts $>7$ nights/month, compared with never night shift works, and ORs, (95\% CIs) were $1.40,(1.12$ to 1.75$)$, 1.56 , (1.24 to 1.97$), 1.57$ (1.24 to 1.98$), 1.36$, (1.06 to $1.74)$ and 1.38 , (1.09 to 1.73$)$, respectively. The results of model 2 showed that the odds of elevated GGT were increased in the groups of the current night shift, the duration of night shifts $>19$ years, the cumulative number of night shifts $>1774$ nights, the average frequency of night shifts $\leq 7$ night/month and the average frequency of night shifts $>7$ nights/month, compared with never night shift works, and ORs, (95\% CIs) were 1.37, (1.09 to 1.73), 
Table 1 Basic characteristics of the study subjects according to current shift status

\begin{tabular}{|c|c|c|c|c|c|}
\hline \multirow[b]{2}{*}{ Characteristics } & \multirow[b]{2}{*}{ Total $(n=7031)$} & \multicolumn{3}{|c|}{ Current shift status } & \multirow[b]{2}{*}{$P$ value } \\
\hline & & Never & Ever & Current & \\
\hline Sex, n (\%) & & & & & $<0.001$ \\
\hline Female & 595 (8.5) & $127(11.0)$ & $134(9.7)$ & $334(7.4)$ & \\
\hline Smoking status, n (\%) & & & & & 0.038 \\
\hline Ever & $375(5.3)$ & $56(4.8)$ & $95(6.9)$ & $224(4.9)$ & \\
\hline Current & $3587(51.1)$ & $574(49.7)$ & $686(49.7)$ & 2327 (51.8) & \\
\hline Drinking status, n (\%) & & & & & 0.805 \\
\hline Never & $4295(61.1)$ & $712(61.6)$ & $833(60.4)$ & $2750(61.2)$ & \\
\hline Ever & $150(2.1)$ & $24(2.1)$ & $35(2.5)$ & $91(2.0)$ & \\
\hline Middle & 695 (9.9) & $120(10.4)$ & $117(8.5)$ & $458(10.2)$ & \\
\hline High & $6206(88.3)$ & $1018(88.1)$ & $1237(89.6)$ & 3951 (87.9) & \\
\hline Sleep duration, n (\%) & & & & & 0.986 \\
\hline$<7$ hours/day & $4446(63.2)$ & $618(53.5)$ & $825(59.8)$ & $3003(66.8)$ & \\
\hline$\geq 7$ hours/day & $2585(36.8)$ & $537(46.5)$ & $555(50.2)$ & 1493 (33.2) & \\
\hline Diabetes, n (\%) & & & & & 0.017 \\
\hline No & 6270 (89.2) & 1007 (87.2) & $1220(88.4)$ & $4043(89.9)$ & \\
\hline Yes & $761(10.8)$ & $148(12.8)$ & $160(11.6)$ & $453(10.1)$ & \\
\hline Renal dysfunction, n (\%) & & & & & 0.898 \\
\hline No & $5923(84.2)$ & $968(83.8)$ & $1162(84.2)$ & $3793(84.4)$ & \\
\hline Yes & 1108 (15.8) & 187 (16.2) & $218(15.8)$ & $703(15.6)$ & \\
\hline BMI $\left(\mathrm{kg} / \mathrm{m}^{2}\right), \mathrm{n}(\%)$ & & & & & 0.426 \\
\hline$<24$ & $2372(33.7)$ & $389(33.7)$ & $466(33.8)$ & $1517(33.7)$ & \\
\hline $24-27$ & $3494(49.7)$ & $563(48.7)$ & 707 (51.2) & $2224(49.5)$ & \\
\hline$\geq 28$ & $1165(16.6)$ & $203(17.6)$ & $207(15.0)$ & 755 (16.8) & \\
\hline High temperature, n (\%) & & & & & $<0.001$ \\
\hline No & 3462 (49.2) & 748 (64.8) & 789 (57.2) & 1925 (42.8) & \\
\hline Yes & 3569 (50.8) & 407 (35.2) & $591(42.8)$ & $2571(57.2)$ & \\
\hline Noise, n (\%) & & & & & 0.602 \\
\hline No & $53(0.8)$ & $6(0.5)$ & $11(0.8)$ & $36(0.8)$ & \\
\hline Yes & 6978 (99.2) & 1149 (99.5) & 1369 (99.2) & 4460 (99.2) & \\
\hline Dust, n (\%) & & & & & $<0.001$ \\
\hline No & 2438 (34.7) & 364 (31.5) & 362 (26.2) & 1712 (38.1) & \\
\hline Yes & 4593 (65.3) & 791 (68.5) & 1018 (73.8) & 2784 (61.9) & \\
\hline CO, n (\%) & & & & & $<0.001$ \\
\hline
\end{tabular}


Table 1 Continued

\begin{tabular}{|c|c|c|c|c|c|}
\hline \multirow[b]{2}{*}{ Characteristics } & \multirow[b]{2}{*}{ Total $(n=7031)$} & \multicolumn{3}{|c|}{ Current shift status } & \multirow[b]{2}{*}{ P value } \\
\hline & & Never & Ever & Current & \\
\hline No & 3906 (55.6) & $550(47.6)$ & $739(53.6)$ & $2617(58.2)$ & \\
\hline \multicolumn{6}{|l|}{ GGT levels, n (\%) } \\
\hline Normal & $6270(89.2)$ & $1057(91.5)$ & $1233(89.3)$ & $3980(88.5)$ & 0.014 \\
\hline \multicolumn{6}{|l|}{ AST levels, n (\%) } \\
\hline Normal & 6846 (97.3) & $1124(97.3)$ & $1339(97.0)$ & $4381(97.4)$ & 0.705 \\
\hline Elevated & $187(2.7)$ & $31(2.7)$ & $41(3.0)$ & $115(2.6)$ & \\
\hline \multicolumn{6}{|l|}{ ALT levels, $n(\%)$} \\
\hline Working years (years), median (range) & $24.4(0.2,46.8)$ & $26.3(0.2,43.1)$ & $26.5(1.4,42.8)$ & $23.8(1.4,46.8)$ & 0.002 \\
\hline DASH Score & $21.6 \pm 2.2$ & $21.7 \pm 2.1$ & $21.7 \pm 2.2$ & $21.6 \pm 2.2$ & 0.856 \\
\hline ALT levels (U/L), median (IQR) & $22(17,31)$ & $22(17,29)$ & $22(17,30)$ & $23(17,31)$ & $<0.001$ \\
\hline AST levels (U/L), median (IQR) & $20(17,23)$ & $20(17,23)$ & $20(17,23)$ & $20(17,23)$ & 0.247 \\
\hline GGT levels (U/L), median (IQR) & $27(19,42)$ & $27(18,40)$ & $26(18,40)$ & $28(19,43)$ & $<0.001$ \\
\hline
\end{tabular}

Values are number of individuals (\%), means \pm SD, and median (range). P-values are from chi-square test for categorical variables and ranksum test for continuous variables.

ALT, alanine transaminase; AST, aspartate aminotransferase; BMI, body mass index; DASH, Dietary Approaches to Stop Hypertension; GGT, gamma-glutamyl transpeptidase; MET, metabolic equivalent of task.;

1.42 , $(1.12$ to 1.80$), 1.41,(1.11$ to 1.80$), 1.32,(1.03$ to $1.70), 1.36,(1.08$ to 1.71$)$, respectively, after adjustment for age and sex. All of the estimates were attenuated but remained significant. The results of model 3 showed that the odds of elevated GGT were increased with the groups of the current night shift, the duration of night shifts $>19$ years, the cumulative number of night shifts $>1774$ nights, the average frequency of night shifts $\leq 7 \mathrm{night} /$ month and the average frequency of night shifts $>7$ nights/month, compared with never night shift workers, and ORs, $(95 \%$ CIs) were 1.39 , (1.10 to 1.75$), 1.46$, (1.15 to 1.86$), 1.46$, (1.15 to 1.85$), 1.34$, (1.04 to 1.73$), 1.37$, (1.09 to 1.74 ), respectively, after adjustment for age, sex, smoking status, drinking status and DASH Score. Though all of the estimates were still attenuated, the results were comparable to model 1. Moreover, our study also showed that the odds of elevated GGT increased with a longer duration of night shifts and a more cumulative number of night shifts. Finally, no significant association was observed between sleep duration and elevated GGT. (table 3) In addition, we also found that the relationship between elevated ALT and different exposure metrics of night shift work were statistically significant (see online supplemental table S3), but this significant association was not found between elevated AST and rotating night shift work (see online supplemental table S4).

The separate effect of shorter sleep duration ( $<7$ hours/ day) on elevated GGT was not statistically significant (table 3), but the separate effects of smoking and drinking status were statistically significant (see online supplemental table S5). And there were no significant interactions between exposure metrics of night shift work and sleep duration on elevated GGT. However, the effect of rotating night shift work on the elevated GGT will be affected by sleep duration. Stratified by sleep duration, it was found that the relationship between rotating night shift work and elevated GGT was statistically significant only in the stratification with shorter sleep duration. And the odds of elevated GGT were increased with the groups of the current night shift, the duration of night shifts $>19$ years, the cumulative number of night shifts $>1774$ nights, compared with never night shift groups, and ORs, (95\% CIs) were 1.40 , (1.03 to 1.92$), 1.41$, (1.02 to 1.94$)$ and $1.40,(1.01$ to 1.93$)$, respectively, after adjusting for potential confounders (table 4).

In addition, we further stratified the analysis using smoking status, drinking status, body mass index (BMI), and physical activity and studied the interaction between rotating night shift work and the lifestyle factors. As shown in table 1 , the number of ever smokers $(2.1 \%)$, ever drinkers $(5.3 \%)$ and high physical activity $(1.8 \%)$ is small. Therefore, we combine ever smokers and never smokers, ever drinkers and never drinkers, and middle and high physical activity into one group, respectively. The association between the rotating night shift work and the elevated GGT in the two layers of current drinking 
Table 2 The distribution of different exposure metrics of night shift work in the study population and according to gamma-glutamyl transpeptidase (GGT) levels

\begin{tabular}{|c|c|c|c|}
\hline \multirow[b]{2}{*}{ Characteristics } & \multicolumn{2}{|c|}{ GGT levels (U/L) } & \multirow[b]{2}{*}{$P$ value } \\
\hline & $\begin{array}{l}\text { Normal } \\
(n=6270)\end{array}$ & $\begin{array}{l}\text { Elevated } \\
(n=761)\end{array}$ & \\
\hline Current shift status, n (\%) & & & 0.014 \\
\hline Never & $1057(16.8)$ & $98(12.8)$ & \\
\hline Ever & $1233(19.7)$ & $147(19.4)$ & \\
\hline Current & $3980(63.5)$ & $516(67.8)$ & \\
\hline Duration of night shifts, $\mathrm{n}(\%)$ & & & $<0.001$ \\
\hline Never & $1057(16.8)$ & $98(12.8)$ & \\
\hline$\leq 19$ years & $2574(41.1)$ & $281(37.0)$ & \\
\hline$>19$ years & $2639(42.1)$ & $382(50.2)$ & \\
\hline Cumulative number of night sh & ifts, n (\%) & & $<0.001$ \\
\hline Never & $1057(16.8)$ & $98(12.8)$ & \\
\hline$\leq 1774$ nights & $2656(42.4)$ & $292(38.4)$ & \\
\hline$>1774$ nights & $2557(40.8)$ & $371(48.8)$ & \\
\hline Average frequency of night shi & fts, n (\%) & & 0.016 \\
\hline Never & $1057(16.8)$ & $98(12.8)$ & \\
\hline$\leq 7$ nights/month & $1893(30.2)$ & $239(31.4)$ & \\
\hline$>7$ nights/month & $3320(53.0)$ & $424(55.8)$ & \\
\hline
\end{tabular}

Values are number of individuals (\%). P values are from $\chi^{2}$ test for categorical variables. The cut-off points of the duration of night shifts, average frequency of night shifts and cumulative number of night shifts were the medians of the corresponding continuous variables.

status was statistically significant (see online supplemental table S6). When we used current smoking status and BMI for stratified analysis, the association between rotating night shift work and elevated GGT was statistically

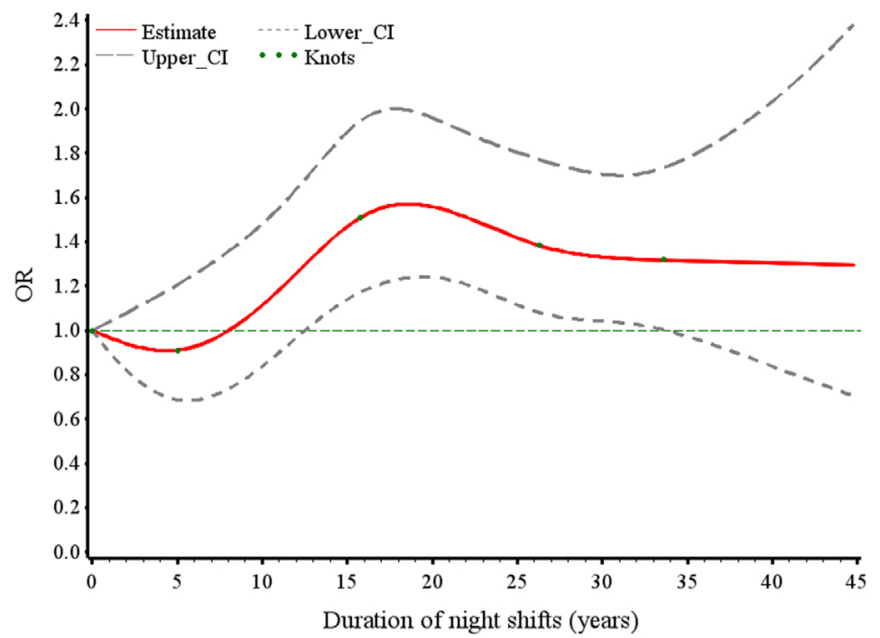

Figure 1 Duration of night shifts (years) and odds of elevated gamma-glutamyl transpeptidase from restricted cubic spline models. The solid lines, the long dashes and the short dashes represent the point estimate of the OR value and the upper and lower limits of its $95 \% \mathrm{Cl}$, respectively. Adjusted for age, sex, smoking status, drinking status and DASH Score. DASH, Dietary Approaches to Stop Hypertension.

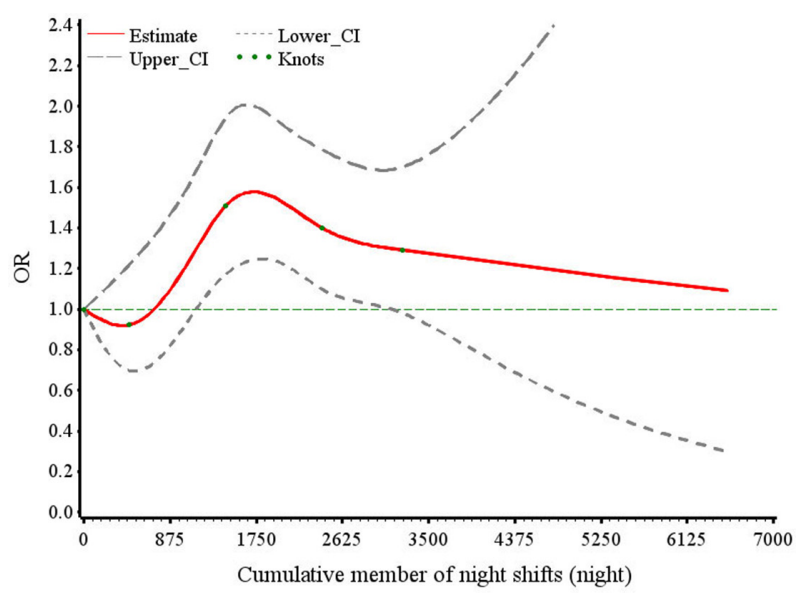

Figure 2 Cumulative member of night shifts (night) and odds of elevated gamma-glutamyl transpeptidase from restricted cubic spline models. The solid lines, the long dashes and the short dashes represent the point estimate of the OR value and the upper and lower limits of its $95 \% \mathrm{Cl}$, respectively. Adjusted for age, sex, smoking status, drinking status and DASH Score. DASH, Dietary Approaches to Stop Hypertension.

significant only in layers with current smoking or BMI $\geq 24$ (see online supplemental tables S7 and S8). The risks of elevated GGT were increased by rotating night shift work in both stratification of physical activity (see online supplemental table S9). In addition, all the interactions between rotating night shift work and lifestyle factors on elevated GGT were not statistically significant.

\section{Sensitivity analyses}

Multivariate logistic regression analysis showed that the results from the population including participants taking antiretroviral drugs were comparable to those excluding participants taking antiretroviral drugs (see online supplemental table S10). In addition, the results of the logistic regression model after adjusting all potential confounding factors were comparable to those of the logistic regression model selected by the stepwise regression method (see online supplemental table S11). In order to avoid the influence of the maximum on the results of RCS curves fitting, we deleted the last $1 \%$ quantile of the duration of night shifts and the cumulative member of night shifts, and the results remained robust (see online supplemental figures S1 and S2).

\section{DISCUSSION}

Previous studies have used duration in rotating night shifts, night shifts per month, cumulative lifetime night shift exposure and other indicators to study the relationship between shift work and other health issues. ${ }^{24}{ }^{25}$ At present, the results of this study showed that all rotating night shift work indicators (current shift status, duration of night shifts, cumulative number of night shifts and average frequency of night shifts) had a significant association with elevated GGT and elevated ALT, but not with 
Table 3 ORs of elevated gamma-glutamyl transpeptidase (GGT) according to different exposure metrics of night shift work

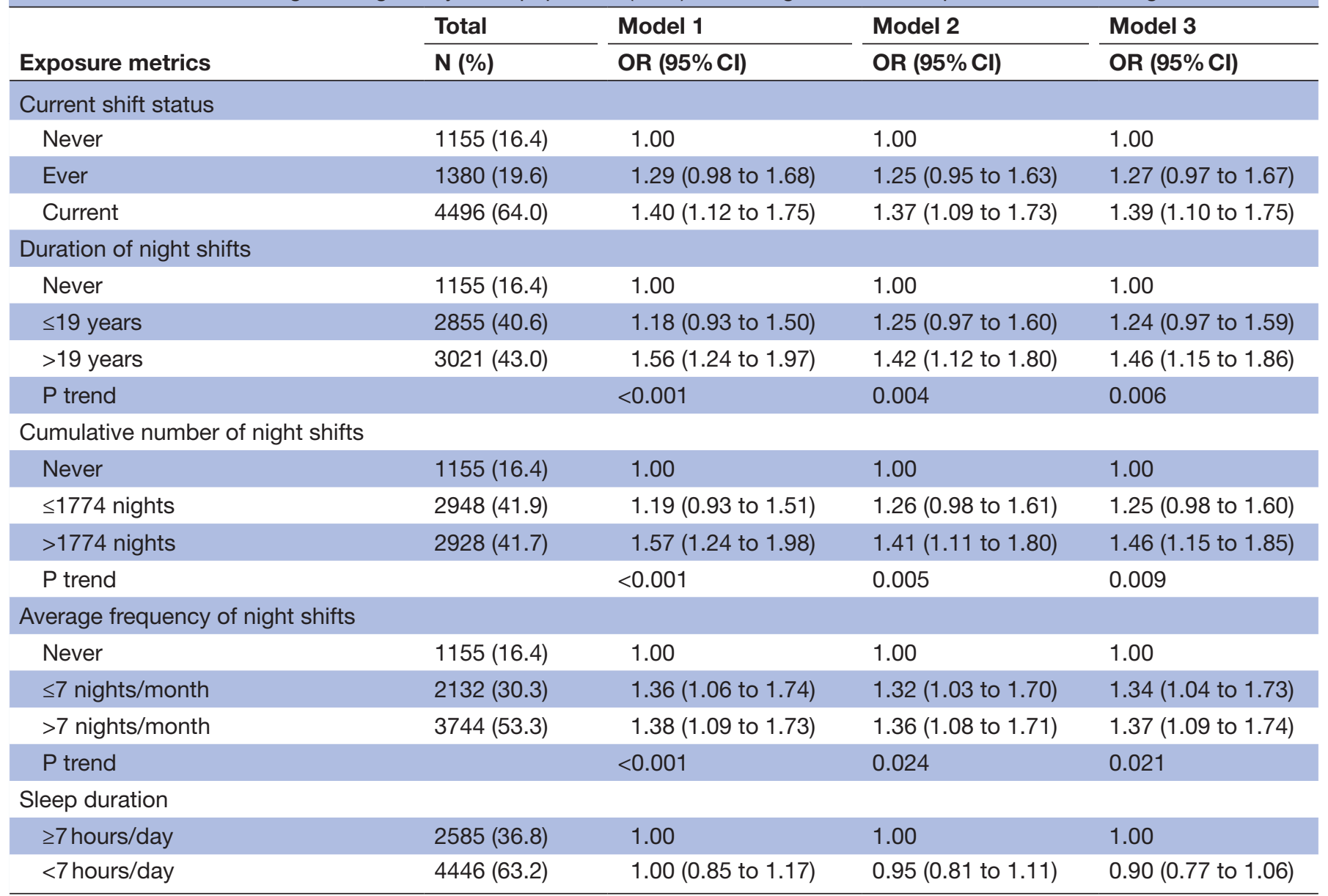

Model 1: unadjusted. Model 2: adjusted for age, sex. Model 3: adjusted for age, sex, smoking status, drinking status and DASH Score. DASH, Dietary Approaches to Stop Hypertension.

elevated AST. The present study indicated that rotating night shift work would increase the risk of elevated GGT and elevated ALT. This is in contradiction with a previous study, which indicated that shift work is not a significant risk factor for increased serum GGT on workers of a telecommunication enterprise. ${ }^{26}$ The difference in exposure assessment and population characteristics may contribute to the discrepancy. Additionally, our study used different indicators to evaluate the rotating night shift work, which reduced the deviation caused by different evaluation standards to a certain extent. However, the results of our study were consistent with some research results to some extent. A study has shown that circadian rhythm disorder is associated with higher levels of GGT, ${ }^{27}$ and some studies have shown that the elevated level of liver enzymes (including ALT, ALP) was significantly associated with shift work. ${ }^{19}{ }^{20}$ Moreover, the results of this study were supported by several previous studies, which indicated that shift work was associated with a higher risk of nonalcoholic fatty liver disease (NAFLD) ${ }^{19}{ }^{28}$ since elevated GGT and elevated ALT is relevant to NAFLD. ${ }^{2930}$ Although the underlying biological pathways have not been fully revealed, some mechanisms have been proposed.
Rotating night shift work participation can cause oxidative stress ${ }^{31}$ and chronic systemic inflammation, ${ }^{32}$ further leading to chronic liver disease. ${ }^{33}$ Elevated GGT is associated with oxidative stress, ${ }^{34}$ and chronic liver disease. ${ }^{35}$ Circadian rhythm plays an important role in the process of regulating related cells, which is related to chronic liver disease. Shift work will affect the regulation process by disturbing the circadian rhythm and causing damage to the liver. ${ }^{36}$ The nutritional metabolism of the liver is regulated by circadian rhythm, and rotating night shift work disrupts the circadian rhythm, resulting in the disorder of liver nutritional metabolism, which has adverse effects on the health of the liver. ${ }^{37}$

Second, our study also showed that the odds of elevated GGT increased with a longer duration of night shifts and a more cumulative number of night shifts. This conclusion has been supported by some studies. This may be related to liver repair, but long-term rotating night shift work will cause adverse effects on the liver which is difficult to repair. It is reported that liver is a highly elastic organ and plays an important role in detoxification. ${ }^{38}$ For example, the levels of GGT will return to normal in a relatively short time after liver repair. ${ }^{39}$ However, long-term 
Table 4 Joint effects of different exposure metrics of night shift work and sleep duration on elevated gamma-glutamyl transpeptidase

\begin{tabular}{|c|c|c|c|c|c|}
\hline \multirow[b]{2}{*}{ Stratified variables } & & Total & Model 1 & Model 2 & Model 3 \\
\hline & & $\mathbf{N}(\%)$ & OR $(95 \% \mathrm{Cl})$ & OR $(95 \% \mathrm{Cl})$ & OR $(95 \% \mathrm{Cl})$ \\
\hline Sleep duration & \multicolumn{5}{|l|}{ Current shift status } \\
\hline \multirow[t]{3}{*}{$<7$ hours/day } & Never & $123(10.1)$ & 1.00 & 1.00 & 1.00 \\
\hline & Ever & $361(14.6)$ & 1.22 (0.85 to 1.75$)$ & 1.20 (0.84 to 1.73$)$ & 1.19 (0.82 to 1.72$)$ \\
\hline & Current & 724 (75.3) & 1.42 (1.04 to 1.92$)$ & 1.41 (1.04 to 1.92$)$ & 1.40 (1.03 to 1.92$)$ \\
\hline \multirow[t]{3}{*}{$\geq 7$ hours/day } & Never & $1032(17.7)$ & 1.00 & 1.00 & 1.00 \\
\hline & Ever & $1771(20.6)$ & 1.39 (0.93 to 2.07$)$ & $1.29(0.86$ to 1.94$)$ & 1.39 (0.92 to 2.09$)$ \\
\hline & Current & $3020(61.7)$ & 1.37 (0.97 to 1.93$)$ & 1.31 (0.93 to 1.85$)$ & 1.37 (0.97 to 1.95$)$ \\
\hline$P$ for interaction & & & 0.720 & 0.520 & 0.686 \\
\hline Sleep duration & \multicolumn{5}{|l|}{ Duration of night shifts } \\
\hline \multirow[t]{3}{*}{$<7$ hours/day } & Never & $123(10.1)$ & 1.00 & 1.00 & 1.00 \\
\hline & $\leq 19$ years & $322(33.7)$ & 1.22 (0.88 to 1.69$)$ & 1.25 (0.89 to 1.75$)$ & $1.27(0.90$ to 1.80$)$ \\
\hline & $>19$ years & $763(56.2)$ & 1.50 (1.10 to 2.05$)$ & $1.44(1.05$ to 1.98$)$ & $1.41(1.02$ to 1.94$)$ \\
\hline \multirow[t]{3}{*}{$\geq 7$ hours/day } & Never & $1032(17.7)$ & 1.00 & 1.00 & 1.00 \\
\hline & $\leq 19$ years & $1951(43.4)$ & 1.12 (0.77 to 1.60$)$ & 1.23 (0.85 to 1.78$)$ & $1.30(0.89$ to 1.90$)$ \\
\hline & $>19$ years & $2840(38.9)$ & 1.74 (1.22 to 2.48$)$ & 1.40 (0.97 to 2.02 ) & 1.46 (1.00 to 2.12$)$ \\
\hline$P$ for interaction & & & 0.338 & 0.448 & 0.289 \\
\hline Sleep duration & $\begin{array}{l}\text { Cumulative number of } \\
\text { night shifts }\end{array}$ & & & & \\
\hline \multirow[t]{3}{*}{$<7$ hours/day } & Never & $123(10.1)$ & 1.00 & 1.00 & 1.00 \\
\hline & $\leq 1774$ nights & $420(34.8)$ & 1.23 (0.89 to 1.70$)$ & 1.25 (0.89 to 1.75$)$ & 1.29 (0.92 to 1.82$)$ \\
\hline & $>1774$ nights & $665(55.1)$ & 1.50 (1.10 to 2.05$)$ & 1.44 (1.05 to 1.98$)$ & $1.40(1.01$ to 1.93$)$ \\
\hline \multirow[t]{3}{*}{$\geq 7$ hours/day } & Never & $1032(17.7)$ & 1.00 & 1.00 & 1.00 \\
\hline & $\leq 1774$ nights & $2528(43.4)$ & $1.26(0.97$ to 1.63$)$ & $1.25(0.86$ to 1.80$)$ & $1.32(0.90$ to 1.91$)$ \\
\hline & $>1774$ nights & $2263(38.9)$ & 1.43 (1.11 to 1.83$)$ & 1.38 (0.95 to 2.00$)$ & $1.45(0.99$ to 2.11$)$ \\
\hline$P$ for interaction & & & 0.351 & 0.452 & 0.267 \\
\hline Sleep duration & $\begin{array}{l}\text { Average frequency of } \\
\text { night shifts }\end{array}$ & & & & \\
\hline \multirow[t]{3}{*}{$<7$ hours/day } & Never & $123(10.1)$ & 1.00 & 1.00 & 1.00 \\
\hline & $\leq 7$ nights/month & 361 (29.9) & 1.37 (0.99 to 1.91$)$ & 1.37 (0.98 to 1.90$)$ & 1.34 (0.96 to 1.88$)$ \\
\hline & $>7$ nights/month & $724(50.0)$ & $1.37(1.01$ to 1.87$)$ & $1.36(1.00$ to 1.86$)$ & $1.36(0.99$ to 1.87$)$ \\
\hline \multirow[t]{3}{*}{$\geq 7$ hours/day } & Never & $1032(17.7)$ & 1.00 & 1.00 & 1.00 \\
\hline & $\leq 7$ nights/month & $1771(30.5)$ & 1.35 (0.93 to 1.96$)$ & 1.25 (0.85 to 1.82$)$ & $1.33(0.91$ to 1.95$)$ \\
\hline & $>7$ nights/month & $3020(51.8)$ & 1.39 (0.98 to 1.97$)$ & 1.35 (0.95 to 1.91$)$ & 1.41 (0.99 to 2.01$)$ \\
\hline$P$ for interaction & & & 0.790 & 0.969 & 0.842 \\
\hline
\end{tabular}

Model 1: unadjusted. Model 2: adjusted for age, sex. Model 3: adjusted for age, sex, smoking status, drinking status and DASH score. DASH, Dietary Approaches to Stop Hypertension.

rotating night shift work will affect liver repair. Lin $e t a t^{40}$ carried out a retrospective analysis on workers who had long-term shift work and found that long-term shift work poses a barrier to ALT normalisation of workers with previous abnormal liver function.

Furthermore, our study showed that the association between the elevated GGT and different exposure metrics of night shift work were statistically significant only when the sleep duration $<7$ hours/day. Kim et $a l^{41}$ has found that shorter sleep duration had progressively higher odds of abnormal ALT. Lack of sleep and poor sleep quality are responsible for a large proportion of the negative effects of shift work, Hicklin and Schwander ${ }^{42}$ said in a study. These studies support the conclusions of this study to some extent, but it needs a lot of studies to confirm.

Finally, we found that current smoking and current drinking status increased the risk of elevated GGT. Moreover, the interactions between rotating night shift work 
and lifestyle factors (smoking, drinking, BMI and physical activity) on elevated GGT were not statistically significant. However, the lifestyle factors affected the association between rotating night shift work and elevated GGT. Among current smokers, people with $\mathrm{BMI} \geq 24$ or people with high physical activity, rotating night shift work would increase the risk of elevated GGT.

In addition, the results of this study are consistent with those of previous studies ${ }^{8}$ to some extent. Rotating night shift work is not only are associated with higher HHcy odds, but also associated with elevated GGT and elevated ALT among steelworkers.

\section{Strengths and limitations}

The first strength of this study is that it has a large sample size of 7031 individuals with detailed disease history, general demographic characteristics and lifestyle information, and we also considered chemical and physical risk factors in the occupational environment. In addition, we have collected in-depth rotating night shift work information for the evaluation of different exposure metrics of night shift work. However, there are some weaknesses of our study. First, this study is a cross-sectional study, which has limitations in explaining the causal relationship of rotating night shift work and sleep duration with elevated GGT. Second, because information on sleep duration and lifestyles was self-reported, the potential exists for exposure misclassification. Thirdly, we have no authority to obtain the specific level of occupational hazards, including high temperature, noise, dust and CO, so we were unable to consider industrial dust in this study. Finally, our research object is relatively healthy steelworkers. Therefore, the results of this study are limited when they are extended to the general population.

\section{CONCLUSION}

In conclusion, our data indicated that current shift status, duration of night shifts, cumulative number of night shifts and average frequency of night shifts were all associated with elevated GGT and elevated ALT among steelworkers, and lifestyle factors affected the association between rotating night shift work and elevated GGT. From the perspective of occupational medical and preventive medicine, the assessment of shift schedules should be more detailed, and night shift workers should ensure enough sleep duration and quit smoking and drinking. We acknowledge the need for prospective cohort studies to confirm that rotating night shift work and these lifestyle factors are harmful to the health of the liver.

Acknowledgements The authors would like to acknowledge all participants and institutions involving the collection of the baseline data.

Contributors QL: Conceptualisation, writing—original draft, definition and was a major contributor in writing the manuscript. SZ and HW: Data curation and investigation. SZ and CX: Methodology. XZ and SQ: Term. JY: Supervision, project administration, funding acquisition and the guarantor. All authors agree to submit this article. All authors read and approved the final manuscript.
Funding This work was supported by the National Key R\&D Program of China (No. 2016YFC0900605).

Competing interests None declared.

Patient consent for publication Not applicable.

Ethics approval This study involves human participants and was approved by The Ethics Committee of North China University of Science and Technology (No.15006). Participants gave informed consent to participate in the study before taking part.

Provenance and peer review Not commissioned; externally peer reviewed.

Data availability statement Data are available upon reasonable request. The datasets generated and analysed during the current study are not publicly available due other analyses are proceeding but are available from the corresponding author on reasonable request.

Supplemental material This content has been supplied by the author(s). It has not been vetted by BMJ Publishing Group Limited (BMJ) and may not have been peer-reviewed. Any opinions or recommendations discussed are solely those of the author(s) and are not endorsed by BMJ. BMJ disclaims all liability and responsibility arising from any reliance placed on the content. Where the content includes any translated material, BMJ does not warrant the accuracy and reliability of the translations (including but not limited to local regulations, clinical guidelines, terminology, drug names and drug dosages), and is not responsible for any error and/or omissions arising from translation and adaptation or otherwise.

Open access This is an open access article distributed in accordance with the Creative Commons Attribution Non Commercial (CC BY-NC 4.0) license, which permits others to distribute, remix, adapt, build upon this work non-commercially, and license their derivative works on different terms, provided the original work is properly cited, appropriate credit is given, any changes made indicated, and the use is non-commercial. See: http://creativecommons.org/licenses/by-nc/4.0/.

ORCID iD

Juxiang Yuan http://orcid.org/0000-0002-7273-2097

\section{REFERENCES}

1 Knutsson A. Methodological aspects of shift-work research. Chronobiol Int 2004;21:1037-47.

2 Buchvold HV, Pallesen S, Waage S, et al. Shift work schedule and night work load: Effects on body mass index-a four-year longitudinal study. Scand J Work Environ Health 2018;44:251-7.

3 Vyas MV, Garg AX, lansavichus AV, et al. Shift work and vascular events: systematic review and meta-analysis. BMJ 2012;345:e4800.

4 Lu L-F, Wang C-P, Tsai I-T, et al. Relationship between shift work and peripheral total and differential leukocyte counts in Chinese steel workers. J Occup Health 2016;58:81-8.

5 Bustamante-Montes LP, Flores-Meza B, Hernández-Valero MA, et al. Night shift work and risk of breast cancer in women. Arch Med Res 2019;50:393-9.

6 Navara KJ, Nelson RJ. The dark side of light at night: physiological, epidemiological, and ecological consequences. J Pineal Res 2007;43:215-24.

7 Fonken LK, Nelson RJ. Illuminating the deleterious effects of light at night. F1000 Med Rep 2011:3-18.

8 Zhang S, Wang Y, Li Q, et al. Different exposure metrics of rotating night shift work and hyperhomocysteinaemia among Chinese steelworkers: a cross-sectional study. BMJ Open 2020;10:e041576.

9 Itani $\mathrm{O}$, Jike M, Watanabe N, et al. Short sleep duration and health outcomes: a systematic review, meta-analysis, and meta-regression. Sleep Med 2017;32:246-56.

10 Tan X, Chapman CD, Cedernaes J, et al. Association between long sleep duration and increased risk of obesity and type 2 diabetes: a review of possible mechanisms. Sleep Med Rev 2018;40:127-34.

11 Altman NG, Izci-Balserak B, Schopfer E, et al. Sleep duration versus sleep insufficiency as predictors of cardiometabolic health outcomes. Sleep Med 2012;13:1261-70.

12 Imaizumi H, Takahashi A, Tanji N, et al. The association between sleep duration and non-alcoholic fatty liver disease among Japanese men and women. Obes Facts 2015;8:234-42.

13 Newsome PN, Cramb R, Davison SM, et al. Guidelines on the management of abnormal liver blood tests. Gut 2018;67:6-19.

14 Agrawal S, Dhiman RK, Limdi JK. Evaluation of abnormal liver function tests. Postgrad Med J 2016;92:223-34.

15 Labayen I, Ruiz JR, Ortega FB, et al. Liver enzymes and clustering cardiometabolic risk factors in European adolescents: the HELENA study. Pediatr Obes 2015;10:361-70. 
16 Zhang J, Cheng N, Ma Y, et al. Liver enzymes, fatty liver and type 2 diabetes mellitus in a Jinchang cohort: a prospective study in adults. Can J Diabetes 2018;42:652-8.

17 Bonham MP, Bonnell EK, Huggins CE. Energy intake of shift workers compared to fixed day workers: a systematic review and metaanalysis. Chronobiol Int 2016;33:1086-100.

18 Cain SW, Filtness AJ, Phillips CL, et al. Enhanced preference for high-fat foods following a simulated night shift. Scand J Work Environ Health 2015;41:288-93.

19 Chen Y, Lauren S, Chang BP, et al. Objective food intake in night and day shift workers: a laboratory study. Clocks Sleep 2018;1:42-9.

20 Wang F, Zhang L, Wu S, et al. Night shift work and abnormal liver function: is non-alcohol fatty liver a necessary mediator? Occup Environ Med 2019;76:83-9.

21 Khosravipour M, Shah Mohammadi M. The effects of exposure to night shift work on liver function: a cross-sectional study with emphasis of alkaline phosphatase enzyme. Chronobiol Int 2020;37:142-5.

22 Ohayon MM, Smolensky MH, Roth T. Consequences of shiftworking on sleep duration, sleepiness, and sleep attacks. Chronobiol Int 2010;27:575-89.

23 Stadler M, Bollow E, Fritsch M, et al. Prevalence of elevated liver enzymes in adults with type 1 diabetes: a multicentre analysis of the German/Austrian DPV database. Diabetes Obes Metab 2017;19:1171-8.

24 Vetter C, Dashti HS, Lane JM, et al. Night shift work, genetic risk, and type 2 diabetes in the UK Biobank. Diabetes Care 2018;41:762-9.

25 Lie J-AS, Kjuus H, Zienolddiny S, et al. Night work and breast cancer risk among Norwegian nurses: assessment by different exposure metrics. Am J Epidemiol 2011;173:1272-9.

26 Higashikawa A, Suwazono Y, Okubo Y, et al. Association of working conditions and lifestyle with increased serum gamma-glutamyltransferase: a follow-up study. Arch Med Res 2005;36:567-73.

27 Rivera-Coll A, Fuentes-Arderiu X, Díez-Noguera A. Circadian rhythms of serum concentrations of 12 enzymes of clinical interest. Chronobiol Int 1993;10:190-200.

28 Verma S, Jensen D, Hart J, et al. Predictive value of ALT levels for non-alcoholic steatohepatitis (NASH) and advanced fibrosis in nonalcoholic fatty liver disease (NAFLD). Liver Int 2013;33:1398-405.
29 Alam S, Noor-E-Alam SM, Chowdhury ZR, et al. Nonalcoholic steatohepatitis in nonalcoholic fatty liver disease patients of Bangladesh. World J Hepatol 2013;5:281-7.

30 Lin Y-C, Chen P-C. Persistent rotating shift work exposure is a tough second hit contributing to abnormal liver function among on-site workers having sonographic fatty liver. Asia Pac J Public Health 2015;27:NP1765-74.

31 Sharifian A, Farahani S, Pasalar P. Shift work as an oxidative stressor. J Circadian Rhythms 2005:3-15.

32 Puttonen S, Viitasalo K, Härmä M. Effect of shiftwork on systemic markers of inflammation. Chronobiol Int 2011;28:528-35.

33 Mandato C, Lucariello S, Licenziati MR, et al. Metabolic, hormonal, oxidative, and inflammatory factors in pediatric obesity-related liver disease. J Pediatr 2005;147:62-6.

34 Sreeram M, Suryakar AN, Dani NH. Is gamma-glutamyl transpeptidase a biomarker for oxidative stress in periodontitis? J Indian Soc Periodontol 2015;19:150-4.

35 Shen Z-W, Xing J, Wang Q-L, et al. Association between serum $\gamma$-glutamyltransferase and chronic kidney disease in urban Han Chinese: a prospective cohort study. Int Urol Nephrol 2017;49:303-12.

36 Mukherji A, Bailey SM, Staels B, et al. The circadian clock and liver function in health and disease. J Hepatol 2019;71:200-11.

37 Barclay JL, Husse J, Bode B, et al. Circadian desynchrony promotes metabolic disruption in a mouse model of shiftwork. PLoS One 2012;7:e37150.

38 Schmid M. Umwelteinfluäusse auf Leber und Magen-Darm-Trakt [Effects of the environment on the liver and gastrointestinal tract]. Schweiz Med Wochenschr 1976;106:289-95.

39 Watanapa P. Recovery patterns of liver function after complete and partial surgical biliary decompression. Am J Surg 1996;171:230-4.

40 Lin Y-C, Hsieh I-C, Chen P-C. Long-term day-and-night rotating shift work poses a barrier to the normalization of alanine transaminase. Chronobiol Int 2014;31:487-95.

$41 \mathrm{Kim}$ D, Kim HJ, Kushida CA, et al. Short sleep duration is associated with abnormal serum aminotransferase activities and nonalcoholic fatty liver disease. Clin Gastroenterol Hepatol 2018;16:588-90.

42 Hicklin D, Schwander J. Schichtarbeit und Schlaf [Shift Work and Sleep]. Praxis 2019;108:119-24. 